\title{
Thyroid microcarcinoma in pediatric patients: how aggressive do we need to be?
}

Melanie Goldfarb

Assistant Professor of Surgery, Endocrine Surgery, John Wayne Cancer Institute/Providence St John's Health Center, 2200 Santa Monica Blvd, Santa Monica, CA 90404, USA

melaniegoldfarbmd@gmail.com

\section{"Our goal as clinicians should be to offer these generally low-risk patients a management strategy with the least morbidity, fewest complications, best long-term cancer control and quality of life."}

Keyw ords: • microcarcinoma • pediatric • thyroid cancer

In adults, papillary thyroid microcarcinoma (PTMC: tumors $\leq 1 \mathrm{~cm}$ ) is now the most common form of thyroid carcinoma [1-4]. In children, TMC is an entity not often discussed since young patients are known to present with more extensive disease and are more often the focus of discussion $[5,6]$. The good news is that the incidence of PTMC in children is decreasing [7]. Nevertheless, given the potential morbidity from thyroid surgery, the potential need for thyroid suppression during important times of growth and development, and long-term quality of life, the management of PTMCs in children needs to be addressed. There are no guidelines or level I studies on the management of these patients. Our goal as clinicians should be to offer these generally low-risk patients a management strategy with the least morbidity, fewest complications, best long-term cancer control and quality of life.

There are two scenarios that lead to the discovery of PTMC: incidental pathological finding when surgery is performed for another reason such as multinodular goiter or Graves' disease and when a less than $1 \mathrm{~cm}$ lesion is discovered through imaging and biopsied preoperatively. Management options arise at the following time points: the decision to biopsy and operate for a less than $1 \mathrm{~cm}$ lesion, what operation to perform, the role of radioiodine (RAI) ablation, TSH suppression and long-term surveillance.

\section{The decision to biopsy. . . \& \& operate}

The proportion of PTMCs that are suspected or discovered prior to surgery is likely much lower in children compared with adults due to the greatly decreased use of neck imaging for medical conditions. Children whose nodules are discovered as a result of imaging will fall into one of three categories: a pediatric cancer survivor with a history of head and neck radiation, a patient with a new neck lump or newly diagnosed hyperthyroidism. For the pediatric cancer survivor, although it is beyond the scope of this editorial to discuss the benefit or harm of screening this population, if an abnormal lesion is discovered on a thyroid ultrasound it should be biopsied, and if suspicious or positive for cancer, removed. Young, secondary thyroid cancer patients have a worse overall survival than young primary thyroid cancer patients, regardless of tumor size [8]. For all other children, since younger patients in general have an increased risk of cancer in less than $1 \mathrm{~cm}$ nodules with abnormal ultrasound features, lesions should be biopsied once they reach a size threshold 7-8 $\mathrm{mm}$. In this population, an abnormal ultrasound finding is more important than increasing size in detecting a nonlatent malignancy $[9,10]$.

In adults, a few recent studies and trials have proposed watching PTMCs with serial ultrasounds [11]. One showed that watching is potentially safe, and if an operation was determined necessary later on due to tumor growth or other factors, recurrence and overall survival were not adversely affected [10]. However, another study with longer follow-up reported somewhat different results [12]. In neither study was age significant for recurrence, though pediatric patients were not included. In children, watchful waiting could mean decades of serial ultrasounds and office visits, which might eventually uncover other lesions, leading to more unnecessary biopsies. It would seem that if thyroid cancer is suspected or discovered in a child, surgery would be the best option for cancer control, relief of anxiety and to decrease the potential multitude of future imaging and biopsies. 


\section{What operation to perform}

In adults, when PTMC is diagnosed preoperatively and is therefore not incidental, the decision to proceed directly with a total thyroidectomy (TTx) is made based on the presence of nodules in the contralateral lobe, abnormal thyroid function or patient preference. Large studies have shown no difference in disease free or overall survival in PTMC patients that received a TTx compared with a lobectomy [13-15]. Moreover, the risks of surgery, including hypoparathyroidism and nerve injury, can be significant, especially in the hands of lower volume surgeons who are performing the majority of operations in the United States in both children and adults [16,17]. Similar reasoning to determine the extent of surgery should therefore be applied in children, though it is important to consider that these patients will have a very long survivorship period including important stages of growth and development after surgery. Additionally, referral to a high volume surgeon for a child with known cancer can help mitigate the risks of surgery.

For both children and adults with TMCs of nonpapillary histology (Hurthle cell, follicular and tallcell) or with extrathyroidal extension, strong consideration should be made for performing a TTx given the increased rate of recurrence and death in these patients [9,18-19]. Male gender and non-White race may also influence survival and therefore influence surgical decisions. The addition of a prophylactic central lymph node dissection (CLND) for TMC in adults is controversial, and should not be undertaken lightly by a surgeon operating on a young patient. The literature is conflicting as to whether the presence of positive central nodes, and therefore the need for a prophylactic CLND, affects recurrence rates and survival in adults; there are no studies on children [14,20-21]. Additionally, the potential complications, albeit rare in the hands of high volume, experienced surgeons, are lifelong and can significantly impact a child's quality of life. Therefore, there does not seem to be an indication at this time to offer a prophylactic CLND upfront to any young patients with a TMC.

\section{To ablate or not to ablate?}

Should the same risk factors in adults be utilized to consider postoperative RAI ablation in these children? Although the thyroid is at its peak of radiosensitivity in these young patients and RAI would thus have the greatest impact, its use in a therapeutic dose must be weighed against the potential long-term side effects: secondary cancers, decreased fertility and salivary dysfunction. Similar to adults, it is possible that a cut-off size of $1 \mathrm{~cm}$ is an arbitrary distinction for TMCs, and therefore the treating clinician should rely on other prognostic fea- tures when considering not only surgical, but possible ablative options as well [22]. A potentially more important detail may be whether the malignancy was known or suspected preoperatively or discovered incidentally on final pathology [23]. In adults, close to $75 \%$ of PTMCs are incidental findings and have a better prognosis than nonincidental cancers [24].

For PTMCs, assuming that a good operation was performed, the benefits do not appear to outweigh the risks of a large RAI dose, even in patients considered to be of intermediate risk due to extrathyroidal extension, nodal metastases or multifocality [25]. The same principal should be applied in children. However, a small ablative dose could be considered, as there are very few long-term effects, especially in patients with an elevated postoperative thyroglobulin $(\mathrm{Tg})$ after $\mathrm{TTx}$, when the clinician knows that thyroid tissue was left behind during TTx, or for intermediate risk tumors, especially TMCs of nonpapillary histology. In the future, molecular markers such as BRAF may help in risk stratification for postsurgical RAI therapy.

\section{TSH suppression}

All TMCs in children should be treated as low risk with respect to TSH suppression and therefore maintained in the low normal range [25]. Children and young adults should be placed on a physiological dose of thyroid hormone and continue close monitoring during all stages of growth and development.

\section{Long-term surveillance}

It is unknown whether patients diagnosed with a TMC require surveillance for only a finite length of time, and if so, what is that length of time. Moreover, the timing and method of monitoring must strike a balance between too much or little screening in order to produce the best outcomes while also being cost-effective and nonanxiety provoking. A reasonable minimum surveillance protocol would follow the ATA recommendations for low-risk patients: baseline ultrasound at 1 year and monitoring of serum $\mathrm{Tg}, \mathrm{Tg}-\mathrm{Ab}$ and TSH every 6-12 months initially, then annually [25]. In patients with intermediate tumor features, consideration for more aggressive surveillance may be warranted for a certain period of time.

\section{Summary}

There is 'support' for both more aggressive and conservative treatment of TMCs in adults. In children, where no literature exists, experience and clinical judgement should guide the management of these patients, though less is probably more in this population. Future studies should address the optimal surgical strategy and longterm surveillance in children with TMCs. 
Financial \& competing interests disclosure

The author has no relevant affiliations or financial involvement with any organization or entity with a financial interest in or financial conflict with the subject matter or materials discussed in the manuscript. This includes employment, consultancies,

\section{References}

1 Enewold L, Zhu K, Ron E et al. Rising thyroid cancer incidence in the United States by demographic and tumor characteristics, 1980-2005. Cancer epidemiology, biomarkers \& prevention : a publication of the American Association for Cancer Research, cosponsored by the American Society of Preventive Oncology. 18(3), 784-791 (2009).

2 Pellegriti G, Frasca F, Regalbuto C, Squatrito S, Vigneri R. Worldwide increasing incidence of thyroid cancer: update on epidemiology and risk factors. J. Cancer Epidemiol. doi:10.1155/2013/965212 (2013) (Epub ahead of print).

3 Hughes DT, Haymart MR, Miller BS, Gauger PG, Doherty GM. The most commonly occurring papillary thyroid cancer in the United States is now a microcarcinoma in a patient older than 45 years. Thyroid 21(3), 231-236 (2011).

4 Rivkees SA, Mazzaferri EL, Verburg FA et al. The treatment of differentiated thyroid cancer in children: emphasis on surgical approach and radioactive iodine therapy. Endocr. Rev. 32(6), 798-826 (2011).

5 Welch Dinauer CA, Tuttle RM, Robie DK et al. Clinical features associated with metastasis and recurrence of differentiated thyroid cancer in children, adolescents and young adults. Clin. Endocrinol. (Oxf). 49(5), 619-628 (1998).

6 Lerner J, Goldfarb M. Pediatric thyroid microcarcinoma. Ann. Surg. Oncol. 21, S96-S97 (2014).

7 Goldfarb M, Freyer DR. Comparison of secondary and primary thyroid cancer in adolescents and young adults. Cancer 120(8), 1155-1161 (2014).

8 Bo YH, Ahn HY, Lee YH et al. Malignancy rate in sonographically suspicious thyroid nodules of less than a centimeter in size does not decrease with decreasing size. $J$. Korean Med. Sci. 26(2), 237-242 (2011).

9 Ito Y, Miyauchi A, Kihara M, Higashiyama T, Kobayashi K, Miya A. Patient age is significantly related to the progression of papillary microcarcinoma of the thyroid under observation. Thyroid 24(1), 27-34 (2014).

10 Ito $\mathrm{Y}$, Uruno $\mathrm{T}$, Nakano $\mathrm{K}$ et al. An observation trial without surgical treatment in patients with papillary microcarcinoma of the thyroid. Thyroid 13(4), 381-387 (2003).

11 Noguchi S, Yamashita H, Uchino S, Watanabe S. Papillary microcarcinoma. World J. Surg. 32(5), 747-753 (2008).

12 Hay ID, Hutchinson ME, Gonzalez-Losada T et al. Papillary thyroid microcarcinoma: a study of 900 cases observed in a 60-year period. Surgery 144(6), 980-987; discussion 987-988 (2008).

13 Lee J, Park JH, Lee CR, Chung WY, Park CS. Long-term outcomes of total thyroidectomy versus thyroid lobectomy for papillary thyroid microcarcinoma: comparative analysis after propensity score matching. Thyroid 23(11), 1408-1415 (2013). honoraria, stock ownership or options, expert testimony, grants or patents received or pending, or royalties.

No writing assistance was utilized in the production of this manuscript.

14 Londero SC, Krogdahl A, Bastholt L et al. Papillary thyroid microcarcinoma in Denmark 1996-2008: a national study of epidemiology and clinical significance. Thyroid 23(9), 1159-1164 (2013).

15 Sosa JA, Bowman HM, Tielsch JM, Powe NR, Gordon TA, Udelsman R. The importance of surgeon experience for clinical and economic outcomes from thyroidectomy. Ann. Surg. 228(3), 320-330 (1998).

16 Tuggle CT, Roman SA, Wang TS et al. Pediatric endocrine surgery: who is operating on our children? Surgery 144(6), 869-877; discussion 877 (2008).

17 Kuo EJ, Goffredo P, Sosa JA, Roman SA. Aggressive variants of papillary thyroid microcarcinoma are associated with extrathyroidal spread and lymph-node metastases: a population-level analysis. Thyroid 23(10), 1305-1311 (2013).

18 Bernstein J, Virk RK, Hui P et al. Tall cell variant of papillary thyroid microcarcinoma: clinicopathologic features with BRAF(V600E) mutational analysis. Thyroid 23(12), 1525-1531 (2013).

19 So YK, Seo MY, Son YI. Prophylactic central lymph node dissection for clinically node-negative papillary thyroid microcarcinoma: influence on serum thyroglobulin level, recurrence rate, and postoperative complications. Surgery 151(2), 192-198 (2012).

20 Caliskan M, Park JH, Jeong JS et al. Role of prophylactic ipsilateral central compartment lymph node dissection in papillary thyroid microcarcinoma. Endocr. J. 59(4), 305-311 (2012).

21 Wartofsky L. Should patients with papillary microcarcinoma undergo radioiodine ablation? Endocrine 44(2), 278-279 (2013).

22 International Agency for Research on Cancer. World Health Organization Classification of Tumours: Pathology and Genetics of Tumours of the Endocrine Organs. Lloyd R, De Lellis R, Heitz P, Eng C (Eds). IARC Press, Lyon, France. (2004).

23 Lombardi CP, Bellantone R, De Crea C et al. Papillary thyroid microcarcinoma: extrathyroidal extension, lymph node metastases, and risk factors for recurrence in a high prevalence of goiter area. World J. Surg. 34(6), 1214-1221 (2010).

$24 \mathrm{Kim}$ HJ, Kim NK, Choi JH et al. Radioactive iodine ablation does not prevent recurrences in patients with papillary thyroid microcarcinoma. Clin. Endocrinol. (Oxf). 78(4), 614-620 (2013).

25 American Thyroid Association Guidelines Taskforce on Thyroid N, Differentiated Thyroid C, Cooper DS et al. Revised American Thyroid Association management guidelines for patients with thyroid nodules and differentiated thyroid cancer. Thyroid 19(11), 1167-1214 (2009). 